\title{
JUVENTUD E IMAGEN «REGIONAL» DEL «MUNDO» Mundo de la vida y sociedad civil
}

\author{
PEDRo CANALES TAPIA* \\ RODRIGO MERINO**
}

\section{INTRODUCCIÓN}

ENTRE EL 25 y 27 de octubre de 2002, en el Centro de Capacitaciones «Ceferino Namuncura» ubicado en la comuna de Padre Las Casas, al sur de Temuco, se llevó a efecto una «Jornada de formación de jóvenes» con el fin de conocer y discutir - entre los participantes- la forma cómo jóvenes de la Araucanía entienden la vida y ordenan el mundo social en el cual les corresponde desenvolverse.

Dicho evento de reflexión, diálogo y conocimiento «con» y «entre» jóvenes de la Novena Región se circunscribió dentro del «Programa de formación de líderes juveniles» impulsado por la Fundación Konrad Adenauer e implementado en esta región por el Instituto de Promoción Agraria (INPROA), en virtud de lazos de cooperación $\mathrm{y}$ fomento mutuo entre ambas instituciones.

Junto a esto cabe destacar, que dentro de un marco general íntimamente ligado con el trabajo junto a Organizaciones de la Sociedad Civil, y por ende, con dirigentes, líderes y potenciales líderes comuni-

* $\quad$ Licenciado en Historia USACH y Magíster en Ciencias Sociales Aplicadas en la UFRO. E-Mail: pedrojuan1@yahoo.es.

** Sociólogo Universidad de La Frontera, Temuco. 
tarios, en nuestra región con énfasis en el segmento mapuche, INPROA ha orientado esta jornada de motivación y animación de líderes que se desenvuelven en un segmento clave en el desarrollo de Chile, como lo es el juvenil; bajo una impronta conceptual que funcionó junto a enunciados y tópicos de lenguaje propios de la dinámica y propuesta diseñado por la Fundación Adenauer, un cúmulo de indicaciones formativas, pedagógicamente recurrentes en el diario accionar de quienes emprenden el camino de liderar y dar sentidos a misiones relevantes dentro de contextos sociales diversos, que activan dos grandes inquietudes entre quienes asumen participar en la jornada: i) por una parte, indagar en propuestas de sentido que ratifiquen concepciones valóricas, o comiencen dicho proceso; y ii) que cuestionen el accionar e inserción de dicho «actuar» en un mundo globalizado y cada día más «virtual». Dicho de otro modo, activar el debate que permita a jóvenes de edades que fluctúan entre los 18 y 25 años puedan reconocerse a sí mismos y reconocer a otros en la diversidad y el respeto por las diferencias.

Los fundamentos que dieron sentido y razón a la jornada de motivación fue sin duda el interés de abrir canales de comunicación entre los diferentes actores sociales y a la necesidad de concertación propia de un sistema relacional que prioriza el trabajo comunitario en la entrega de reconocimiento jurídico, distribución de recursos y mecanismos de control y fiscalización intervinientes.

Oscar Dávila León sostiene que la órbita juvenil en Chile, en su reconocimiento y dinámica, ha resultado ser un segmento de la población al cual el Estado no atribuye una especificidad en sí misma, sino que en cuanto parte de alguna institucionalidad «aceptada» por el poder. Esta expresión se observa cuando plantea:

El diseño de políticas desde el Estado, ha tenido una directa relación con los grados de visibilidad de los sectores y/o actores sociales en la escena pública. Por ello no es novedad el concebir a lo juvenil — desde una mirada sectorial- como formando parte del sistema educacional, o en su única calidad de estudiantes; por lo que una política hacia ese sector pasaba por la ejecución de políticas en lo educacional. Eso se verifica desde principios de siglo con el aumento en la cobertura y ampliación de la matrícula escolar; y con más fuerza a partir de las décadas de los 50 y 60 con la ampliación de la educación universitaria y el acceso a ella de nuevos sectores sociales provenientes de la pujante clase media chilena. ${ }^{1}$

1 Oscar Dávila León (2000): «Política pública e institucionalidad en juventud». Última Década Nº12. Viña del Mar: Ediciones CIDPA. 
Desde la historia, la problemática estudiada presenta la juventud como parte de la sociedad chilena, dominada durante el siglo XIX por el influjo europeo, la tendencia cortoplacista de sus empresarios y una impronta excluyente definitoria del Estado. El reflejo más claro, a pesar de la proliferación de escuelas y liceos, la educación.

En este orden de cosas, cabe mencionar como objetivo central de esta experiencia, la posibilidad de dialogar e intercambiar opiniones entre los jóvenes y los líderes convocados a este encuentro, con el fin de despertar en ellos —o fortalecer si así fuere- sus deseos de servicio público y su orientar sus afanes dirigenciales por la senda conductual, semántica y propositiva ad hoc con un contexto difícil y hostil a la eclosión de proyectos gregarios de orden público.

Para conseguir dichas metas no bastó con desplegar una actividad y punto. No. Se debió generar una metodología de trabajo y reunión capaz de motivar e impulsar a los jóvenes a comprometer su vida con una o diversas causas que más que nobles deben marcar la pauta de lo que significa servir en lo público y privado, iniciado el siglo XXI. En este sentido, el universo considerado ascendió a 16 jóvenes de entre 18 y 25 años venidos de todos los puntos de la Araucanía; la recolección de información se realizó por medio de Grupos Focales, analizando el sentido discursivo contenido en la oralidad de los jóvenes. El desafío es enorme, que duda cabe, sin embargo, los lineamientos aquí señalados, más la promoción de valores como la justicia, la solidaridad y la probidad resultan de suyo hitos alentadores hoy por hoy.

\section{MARCO TEÓRICO-CONCEPTUAL}

Pues bien, es ahí donde asume un rol preponderante la idea de mundo de la vida ${ }^{2}$ por cuanto marca y encausa el desenvolvimiento y la reflexión constante entre sujetos que interactuando en un mundo cada vez más conectado vía medios de comunicación masivos y la diligencia de la lógica web, deben comprender que la esencia de las relaciones sociales y el trabajo comunitario ya no es más al amparo del Estado otrora benefactor o asistencialista, sino que cada día más preocupado de la regulación de las relaciones socioeconómicas entre el sector privado y la sociedad civil consumidora. No es de extrañar entonces, que uno de los grandes pasos del actual gobierno sea precisamente modernizar la gestión estatal fomentando un nuevo trato con la sociedad civil, con los ciudadanos.

2 Fundación Konrad Adenauer (2002): Documento de Trabajo. 
En este modelo mundo de la vida, las directrices centrales relucen de la propia dinámica del ser histórico de la sociedad. Esto quiere decir, que las dos coordenadas básicas de este esquema son el tiempo histórico y el espacio. Sin duda, que de esta suerte de interacción dialéctica entre dichas expresiones, surge la particularidad de cada complejo social. Por motivos didácticos, dicha figura para los efectos de esta jornada, será entendida desde dos grandes nódulos: un nivel general o mundo y otro nivel particular o país. Así, el desenvolvimiento social de los individuos cobra sentido a partir del contacto y la construcción nómica que cada uno de los actores sociales articula en su interrelación — también- con otros sujetos. No resulta paradójico entonces encontrar jóvenes que opinan estar o sentirse bien en el mundo, pero no en Chile o viceversa. El tema de las valoraciones y experiencias personales o grupales son disímiles, complejas y exteriorizadas desde cuerpos semántico que marcan la voz en un aspecto más que en otro dependiendo de un entramado global específico.

Ahora bien, de esta reflexión surgen otros aspectos relevantes sobre este mundo de la vida. Por una parte, las coordenadas puntuales que se extrapolan del tiempo y el espacio. Por una lado, la coordenada pasado-porvenir, cargada de antecedentes y marcas vivenciales que se proyectan en la intervención subjetiva en las agrupaciones en las cuales se participa y construye ciudadanía, o en espacio privados como la familia. Por otra parte, la coordenada público-privado, también relacionada con el espacio y la misión que cada cual desea o pretende desempeñar en este marco general.

Este esquema denominado de las «4 Ps», permite a su vez, vislumbrar, describir y analizar los diferentes espacios o, como se señala en esta presentación, los mundos del sujeto, comprendidos por el mundo de las utopías, de los proyectos de vida, de la historia común y de las vivencias personales. Sin embargo, en esta estructura conceptual, los anhelos y el aliento de líderes juveniles no pueden quedar sólo en el papel y su opinión en plenarios. Sin sustraer valor alguno a estas posibilidades, resulta urgente preparar o robustecer el acerbo semántico-técnico inclusive, de los jóvenes que lideran o lideraran a agrupaciones de base. Por este motivo, conceptos como participación ciudadana, sociedad civil y nuevo trato entre el Estado y las organizaciones de la sociedad civil, entre la sociedad en general y los pueblos originarios, resultan entre otras, tópicos gravitantes y definitorios de la jornada en cuestión. 
¿Por qué esta orientación? Sin duda alguna, producto de una innata relación entre trabajo en organizaciones sociales, humanismo cristiano y el mundo de la vida. Se trata de conversar en torno a los compromisos de vida asumidos por la juventud dirigente, el rol asignado en dicha opción de las directrices de este humanismo y empapar a estos jóvenes con términos, conceptos y situaciones propias del diario vivir como líderes. Sólo por medio de estos cauces, la historia común, las vivencias personales, utopías y proyectos de vida, entre otros, cobran genuino cuerpo. La sociedad civil construye actualmente sus espacios; el Estado permite, controlando y fiscalizando la ocupación de los recursos por éste asignado a las organizaciones sociales — siempre fue así, e incluso más; en el siglo decimonónico, el Estado creó, o ayudó a hacerlo, a la sociedad civil- . A su vez, en un contexto de nuevo trato, definido - a grosso modo- como la convicción del Estado de entregarle más poder a las organizaciones comunitarias y por tanto concederles la posibilidad de acrecentar la autogestión local y la definición de los «propios destinos». Aquí resulta vital, la participación ciudadana y su grado de posicionamiento de sus roles y atribuciones resguardando la integridad y cumplimiento de los deberes, tantas veces olvidados al fragor de la lucha y las justas reivindicaciones. Resulta imposible negar, la mutua y entrañable relación entonces entre este mundo de la vida anteriormente definido y los temas relativos a la sociedad civil en cuando hábitat social y político de los líderes juveniles. ${ }^{3}$

\section{ESTRATEGIA METODOLÓGICA}

Cuatro partes, todas ellas conectadas entre sí, resultan ser la columna vertebral de la estrategia metodológica asumida. En primer lugar, una dinámica de presentación: el objetivo de esta dinámica fue la presentación y facilitación del conocimiento entre los participantes, además de la indagación con respecto al por qué de la asistencia al taller (motivación y expectativas). En segundo lugar, la mencionada técnica de grupo focal, definida como una entrevista a varias personas a la vez; los participantes conversan unos con otros bajo la guía de un moderador o monitor con el propósito de generar ideas e información

3 Jürgen Habermas (1990): La lógica de las ciencias sociales. Madrid: Tecnos. Platea el autor que desde las ciencias sociales los sujetos deben ser estudiados desde sus definiciones, considerando contextos y una relación entre teoría-práctica. 
importante sobre el tema en cuestión. En tercer lugar, el criterio de selección de grupos más apropiado para esta eventualidad fue el de homogeneidad etárea y de liderato. Por último, los plenarios. En ese momento se ha de reunir la totalidad de los asistentes a la jornada, y a partir del trabajo realizado por cada grupo focal un representante de cada grupo se refiere a los principales planteamientos que en conjunto elaboró en función del tema aludido.

\section{a) Los talleres juveniles: hablemos del primero}

El primer tema de la jornada fue titulado «el mundo de la vida... La lógica de las 4 Ps», cuyo lema fue sencillamente «el mundo de la vida». ${ }^{4}$ En este taller el objetivo proyectado fue conseguir que los jóvenes pudiese comunicarse desde el mundo de la vida «personal» al mundo de la vida «social, histórico y espacial». La pregunta ordenadora del debate fue ¿cómo me siento en el mundo y en el país que me toca vivir? La idea era comenzar a conversar.

Para abordar esta consulta, resulta fundamental entender el concepto de «mundo de la vida». Para Alfred Schutz «el mundo de la vida es toda la esfera de las experiencias, orientaciones y acciones cotidianas, a través de los cuales los individuos persiguen sus intereses y sus asuntos, manipulando objetos, tratando con personas, concibiendo planes y llevándolos a cabo». Para actuar, nos ubicamos en el mundo de la vida. Hay algo así como una «brújula humana» que nos permite ubicarnos en el mundo de la vida. Esa brújula tiene dos grandes ejes: i) un eje en el tiempo; y ii) un eje en el espacio social.

Para situarse en el mundo de la vida debemos trabajar en grupo las coordenadas básicas: i) el eje público-privado; y ii) el eje porvenir-pasado. Si nos situamos en el escenario trazado por los dos ejes y las cuatro «Ps» surgen «los mundos del sujeto»: la historia común (memoria colectiva), las utopías, las vivencias personales y los proyectos de vida. El mundo de la vida es compartido; se pone en común que tenemos de ese mundo: i) dónde estamos; y ii) hacia dónde quisiéramos ir.

Para compartir las visiones y las metas que tenemos sobre el mundo y el país en que nos toca vivir, es necesario ponerse de acuerdo en cómo hacer el discurso dirigido a otros con nuestras visiones y nuestras metas. Ese discurso tendrá contenidos proposicionales (temas

$4 \quad$ Fundación Konrad Adenauer: op. cit. 
y planes expresados en el habla) y contenidos ilocucionarios (la fuerza y convicción del lenguaje de nuestra habla). El discurso que construimos en el grupo es un proyecto común en torno al cual hemos negociado nuestros puntos de vista individuales. La creatividad desplegada en esa negociación es el factor que nos pone en la vanguardia: los que tienen el empuje (la motivación) para empezar el recorrido y la aventura de realización del proyecto común.

\section{b) Procesos que dan coherencia al mundo de la vida}

Como primer proceso: «el paso de la esfera del pasado al porvenir. Este proceso se llama la IDENTIDAD». El segundo proceso: «el paso de la esfera privada a la esfera pública. Este proceso se llama PARTICIPACIÓN». Por su parte, la identidad vincula la historia común con las utopías; relaciona las coyunturas históricas con los movimientos sociales; vincula las vivencias personales con los proyectos de vida; y conecta las experiencias del gozo y el dolor con el desarrollo personal. A su vez, la participación vincula las vivencias personales con la historia común; relaciona las experiencias del gozo y del dolor con las coyunturas históricas; vincula los proyectos de vida con las utopías; y relaciona el desarrollo personal con los movimientos sociales.

Las respuestas entregadas por los jóvenes acerca de la consulta ¿cómo me siento en el mundo y país que me toca vivir?, pueden ser resumidas de la siguiente forma.

\footnotetext{
- $\quad$ Me siento bien de haber nacido en Chile.

- $\quad$ Me gusta Chile por su estabilidad social y económica.

- Casi conforme; logro objetivos, pero falta mayor consideración con los jóvenes.

- $\quad$ Faltan oportunidades de todo tipo; faltan espacios.

- Un poco marginado en el ámbito social como mapuche.

- $\quad$ Extraño, por que por todo lo que se luchó por años, ahora se desprecia.

- $\quad$ Poco conforme con el mundo y con Chile (por su idiosincrasia y «doble estándar»).

- $\quad$ Mal por falta de presencia juvenil en el mundo de las decisiones.

- $\quad$ Falta libertad de expresión.

- $\quad$ Carencia de compromiso real con el desarrollo de Chile.

- Desvalorización y estereotipación juvenil.

- $\quad$ El mundo desilusiona.

- $\quad$ Como no he tenido problemas, no me siento mal; todo lo contrario.
} 
- $\quad$ Falta apoyar actividades de aprendizaje y formación ciudadana.

- A pesar de todos los problemas, me gusta mi país, pues ha salido adelante.

Los jóvenes en general, y especialmente las jóvenes del grupo, manifestaron en mayor cantidad de ocasiones sentirse bien con el país en el cual les toca vivir; no así los varones; esta indicación puede cobrar sentido interpretativo cuando localizamos ciertos hitos históricos propios del comportamiento social en regiones aún fuertemente conectadas con la vida rural y sus tradiciones. En este sentido, los hombres pueden considerar que el mundo y Chile son lugares en los cuales no existe mucho espacio y oportunidades sociales, laborales, etc., pues las exigencias sociales se lo están planteando a diario; no así a las mujeres, cuestión cada día más relativa, sin embargo, puesto que la inserción de la mujer a la vida laboral, por ejemplo, ha ido incrementándose fuertemente en las últimas décadas, no obstante, no tener el problema de la presión y el «qué dirán» si permanece en su casa.

En otro sentido, si bien la pregunta aludía al mundo y a Chile, en su gran mayoría las respuestas aludieron más al país que al mundo en su conjunto; dicho de otra manera y recordando las coordenadas tiempo-espacio, la juventud signa fuertemente la realidad local y regional como «la» realidad en sí. Es por ello, que los comentarios locales y mundiales «significaban» asuntos análogos a la hora del debate y el compartir experiencias.

La segunda consulta que orientó la discusión asumió una postura que adentraba a los compromisos personales: ¿cómo debo actuar de acuerdo a ello? Las respuestas fueron diversas:

- $\quad$ Hacer valer mis derechos.

- $\quad$ Actuar con estima y esfuerzo en pos de objetivos claros.

- $\quad$ Darme a conocer, aportar ideas y proyecciones.

- No «dejarme estar», no sucumbir ante las «inclemencias» del momento.

- Siendo perseverante, no claudicando.

- $\quad$ Con optimismo y esfuerzo; trazando objetivos y cumpliéndolos («sembrar, cuidar y cosechar lo sembrado»).

- $\quad$ Pensando positivamente.

- Como falta participación e información, luchar por estos espacios.

- Dar opiniones y que éstas se transformen algún día en hechos.

- Con respeto y buscando el conocimiento.

- $\quad$ Escuchando a los antiguos y aprender de ellos.

- Impulsando acciones que apoyen el desarrollo integral de los individuos y la sociedad en su conjunto. 
La mayoría de los jóvenes en sus respuesta, asumieron como posturas elementales para actuar en el «mundo y el país que les corresponde vivir», elementos actitudinales positivos, optimistas y de laboriosidad. «Sacar a adelante con esfuerzo y ganas a Chile de sus problemas» fue la consigna. Sin embargo, hubo ciertos matices. Por ejemplo, hubo quienes reconocieron tener talentos en labores de liderato y por tanto consideraron imprescindible «presentarse» ante los órganos correspondientes para colaborar con el desarrollo del país. Otros, a su vez, reflejaron su interés por instruirse antes de intervenir y participar derechamente en acciones dirigenciales: «Buscar el conocimiento» se dijo. Y hubo quienes, asumieron posturas más técnicojurídicas, pues sostuvieron que defendiendo y exigiendo el cumplimiento de los derechos ciudadanos podría revertirse la situación de indefensión, marginación y falta de oportunidades que afectan a los jóvenes.

\section{c) Acerca del segundo taller}

El segundo tema se denominó «Participación ciudadana y sociedad civil: utopías y proyectos de vida»; cuyo lema fue «Participar en nuestra ley». ${ }^{5}$ En este taller el objetivo perseguido fue articular un espacio en el cual los líderes juveniles debatiesen y acordaran —aun en el disenso- formular observaciones o sugerencias a las propuestas legislativas impulsadas en todo Chile por la División de Organizaciones Sociales (DOS) del Ministerio Secretaría General de Gobierno, a través de talleres comunales de discusión que permitan crear un marco legal que fortalezca y entregue herramientas de trabajo y acción a las Organizaciones de la Sociedad Civil (OSCs). ${ }^{6}$

Para los efectos del buen desarrollo del taller, jóvenes y monitores iniciaron la actividad aclarando dudas e inquietudes acerca de la temática abordada. Así por ejemplo, el concepto de sociedad civil fue definida para los efectos de la conversación, como la totalidad de los

5 Jessica Rebolledo y Andrea Sáez Jara (2002): «Manual monitor. Talleres comunales y encuentro regional 'participar en nuestra ley'». Temuco: DOS, BID e INPROA.

6 Juan Podestá Arzubiaga (2001): «Problematización de las políticas públicas desde la óptica regional». Última Década Nº15. Viña del Mar: Ediciones CIDPA. Dice: «la tarea crucial es articular el Estado como representación del bien colectivo y depositario de los recursos financieros provistos por todos los habitantes», p. 166. 
ciudadanos, asociaciones y organizaciones que se agrupan por intereses, valores y anhelos muy diversos. En un sentido restringido, sin embargo, la sociedad civil — debemos aclarar — alude al conjunto de asociaciones y organizaciones privadas sin fines de lucro que persiguen fines sociales de bienestar e interés público.

En este contexto ¿cuál es el rol del Estado? En síntesis, cuatro roles: i) Función articuladora: posibilita construir acuerdos entre actores diversos del país; ii) Función reguladora: abocada a garantizar el ordenamiento y equilibrio de los intereses de los distintos sectores en función del bien común; iii) Traspaso real de poder: a través de la creación de espacios concretos de participación ciudadana; y iv) Modernización del Estado: paso clave en el fortalecimiento de la sociedad civil. Apunta fundamentalmente a asegurar el eficiente uso de recursos públicos, descentralización de las decisiones y apertura a la creación de una cultura de redes organizacionales. En otras palabras, ciudadanizar la gestión de las políticas públicas.

$\dot{¿}$ Y el rol del sector privado? Fundamentalmente ser aliado en la estrategia y contacto Estado/sociedad civil. Colaborar con las OSCs por medio de recursos que apunten a desarrollar acciones de interés público.

En este sentido la DOS, llamó entre octubre y diciembre del año 2002 a consulta ciudadana a todos los dirigentes comunitarios, corporaciones y fundaciones con el fin de aportar a una nueva legislación que fortaleciera a dichas OsCs. Los jóvenes congregados en Padre las Casas también participaron de dicho proceso. Comenzando la discusión, conociendo los niveles de intervención legislativa acerca de las OSCs: i) El status general de las OsCs en el ordenamiento constitucional; ii) Reconocimiento de determinadas OsCs portadoras y promotoras de ciertos intereses públicos; iii) Reconocimiento de la función de las OSCs específicas, tradicionalmente existente en la base territorial (juntas de vecinos y demás organizaciones comunitarias); iv) $\mathrm{La}$ definición de instrumentos específicos institucionales de financiamiento de la acción filantrópica, solidaria o de interés público de las OSCs (fondos concursables, leyes de aportes y subvenciones); y v) El reconocimiento del derecho a participar y la institucionalización de mecanismos de participación ciudadana de las OsCs.

Luego del segundo grupo focal se llevó a cabo la presentación de sugerencias a las propuestas legislativas tendientes a fortalecer la sociedad civil y las organizaciones sociales. Los planteamientos juveniles a propósito de esta discusión, pueden ser sintetizados mediante las siguientes quince propuestas. 
i) Capacitación permanente de líderes de oscs. Escuela para dirigentes y líderes sociales.

ii) Crear oficina de asesoría para las oscs en cada municipio.

iii) Establecer un mínimo de socios según organización y objetivos (funcionales y/o territoriales).

iv) Establecer edad mínima para integrantes de oscs. En caso de menores de edad con autorización y control de un apoderado.

v) Crear organismo que regule actividades de las oscs. Superintendencia de oscs para los temas de recursos y una oficina gubernamental para la línea participación.

vi) Actos de reconocimiento público (en plazas, parques, teatros, colegios, con invitación a medios de comunicación) a las oscs destacadas o sobresalientes en algún aspecto (mínimo una vez al año).

vii) Creación de oficina que permita hacer valer derechos de participación y conocer deberes ciudadanos y de las oscs.

viii) Crear organismos de información ciudadana abierto y de fácil acceso (no sólo infocentros).

ix) Crear confederaciones de oscs que puedan participar con derecho a voz en sesiones del CORE.

x) Crear mecanismo mixto de votación de autoridades regionales y provinciales (con incidencia presidencia y ciudadana).

xi) Actualizar leyes relativas a oscs de forma más expedita y flexible (sin dilación ni demora: los tiempos cambian rápidamente, las leyes y sus modificaciones también).

xii) Crear un organismo fiscalizador de fondos mixtos concursables.

xiii) Información actualizada y pormenorizada de destinos y ocupaciones del fondo mixto.

xiv) Montos graduados concursables fondo mixto (nivel municipal, regional y nacional dependiendo del sentido y alcance del proyecto).

xv) Permitir la repostulación de proyectos previas enmiendas, debidamente informadas a los dirigentes de las OsCs.

Conocidas algunas de las propuesta legislativas impulsadas por la DOS, los jóvenes se dispusieron a trabajar junto al monitor a cargo de los aspectos temático-metodológicos, dentro de los cánones propios de un grupo focal. En esta instancia, la participación libre, espontánea y permanente fue sin duda un elemento alentador de la discusión y el franco debate. A grandes rasgos, las sugerencias juveniles a la propuesta de la DOS apuntaron hacia algunos aspectos que a continuación se consignan: creación de organismos que apoyen e institucionalicen el trabajo de las OsCs; fomento a toda forma de conocimiento y control de los fondos concursables en los cuales puedan participar las oscs; fortalecer la labor fiscalizadora del Estado; participar 
las oscs en espacios de decisión vetados hoy (CORE) y flexibilizar, en torno a la constitución de organizaciones sociales, el número y la edad de sus miembros.

Para Víctor Guerrero Cossio, la diversificación de la sociedad y la fragmentación de las políticas, ha generado un cuadro social:

...que se confronta con los dos enfoques del desarrollo predominantes en el mundo: el de focalización y el de universalización, el primero por enfatizar la atención hacia los grupos extremadamente débiles y el segundo por tender a soluciones totalizantes, que chocan con la naturaleza más particularizada de la realidad social. ${ }^{7}$

Esta pauta implica necesariamente detener la observación en la jerarquización valórica y las prioridades del mundo juvenil respeto de su labor como líderes y el de las actuales autoridades. La defensa de la trasparencia, y su fomento, fueron sin duda la nota alta en dicho ejercicio dialogal. Otro aspecto a resaltar fue la imperiosa necesidad de crear espacios de formación y capacitación constante para dirigentes sociales. No hacerlo, creen los jóvenes, es estar varios pasos atrás respecto de la sociedad privada y el Estado. Por último, en este ámbito analítico, los jóvenes no quieren ser presa de la exclusión o la falta de oportunidades. Dijeron ser el futuro, no obstante, con el transcurso de la sesión, giraron su convicción, expresando ser el «hoy», un presente en expansión.

\section{d) Análisis del desarrollo de la experiencia}

Ámbito operativo-administrativo: el trabajo resultó grato en términos infraestructurales, pues, el centro en el cual se desarrolló reunía los requisitos indispensables como para que las sesiones grupales, plenarios y momentos de conversación resultaran lo más ameno y convenientemente posible.

Ámbito temático-metodológico: los objetivos de la jornada fueron siendo cumplidos momento a momento. Los jóvenes entendieron el mensaje respeto del mundo de la vida, cuestión aplicada con crecer al momento de sugerir alternativas a la propuesta legislativa antes mencionada. La metodología de acción se basó en la acción comunicativa, «los individuos construyen su mundo a través de la

7 Víctor Guerrero Cossio (2001): «Los sujetos de la nueva política social». Última Década Nº15. Viña del Mar: Ediciones CIDPA, p. 186. 
comunicación», y la técnica utilizada, entrevista en grupo focal, fue reconocida como simple y posible de realizar por los distintos sujetos asistentes a la jornada.

La oralidad juvenil no ha sido del todo considerada en estudios de sociología o historia hasta el momento. Casi siempre se mira en Chile esta posibilidad cercana los pueblos indígenas, perdiendo una oportunidad cierta de construir conocimiento de los jóvenes que respalde propuestas de promoción juvenil. Estas políticas:

...la segunda mitad de la década de los noventa representó prácticamente un estancamiento y — de a momentos - un retroceso en lo poco andado en materia de política pública de juventud, en comparación a la primer mitad de la década. Y no es que haya sido mucho el avance en aquélla, sino que más bien estuvo marcada por los intentos de construir e implementar una política de juventud, esfuerzos que estuvieron más en el plano de posicionar «lo juvenil» en la agenda pública, a través de algunos principios e instrumentos, preferentemente programáticos y con una meridiana claridad en los énfasis necesarios de articular y las falencias a superar. ${ }^{8}$

Los factores que explican dichos frenos son entendidos por Oscar Dávila de la siguiente forma:

A modo de enunciación, no podemos dejar de lado dos factores, de diferente naturaleza, pero con consecuencias más o menos similares: la escasa preocupación del tema juvenil como política pública; y la crisis sufrida por el Instituto Nacional de la Juventud (INJ) durante 1997, lo que fuera de poner en abierto cuestionamiento a este servicio, restó credibilidad pública tanto al servicio en cuestión, como a un quehacer centrado en el sector juvenil. Incluso se estuvo cercano a eliminar dicho servicio, no habiendo defensas a su quehacer, ni políticas ni técnicas.

Estos dos factores hicieron retrotraer el tema de políticas de juventud al estado en que se estaba al comienzo de la década, fundamentalmente por la premisa de que no es dable pensar en avanzar en política de juventud, si no se avanza en la instalación de una institucionalidad en materia de juventud. ${ }^{9}$

Esto sin duda es el motivo que debe impulsar a los cientistas sociales a crear espacios de discusión juvenil, pero no sólo eso. Junto con ser marcos metodológicos serios, coherentes, factibles y atingentes a la situación estudiada, deben ser esquemas que busquen activar quiebres con los nodos organizacionales propios de la modernidad, en cuanto articuladores de discursos casi siempre desde la vereda del

8 Dávila: op. cit.

9 Dávila: op. cit. 
Estado o los grupos de poder. Con esto se quiere plantear, no una subversión desde las ciencias, sino que un compromiso genuino con los proyectos y utopías juveniles.

\section{CONCLUSIONES Y PERSPECTIVAS QUE ABRE EL TRABAJO}

Antes de concluir cabe consignar que como imagen de la juventud respecto de la región y el mundo, es necesario mantener un seguimiento de los participantes en actividades de este tipo. Habitualmente, luego de estos encuentros, en los cuales pareciera más la muestra y el registro de los participantes, las conclusiones y la discusión eclosionada de los talleres se dispersa u olvida en anales por muy poca gente consultados. Como sostiene Juan Podestá Arzubiaga, acerca de la confesión de políticas públicas desde una perspectiva regional,

Hay que abrir un espacio de discusión sobre el impacto de las políticas públicas en la dimensión territorial, es decir, qué tipo de políticas debe aplicarse en el ámbito nacional, cuáles en el nivel regional, así como las especificidades de las políticas en el ámbito comunal y local. ${ }^{10}$

Pareciera entonces que la conectividad es sin duda uno de los grandes «talones de Aquiles» de la Araucanía; quizás detrás de este retraso se encubra algún complejo de país o la visión histórica que el Estado tuvo de la Araucanía como espacio cerealero y nada más, obviando la presencia mapuche, de colonos chilenos y foráneos y otros antecedentes que al no ser considerados no fueron analizados en su integridad y situación coyuntural.

Además, se deben utilizar nociones constructivista en la formulación de las sesiones, como medio de asegurar mayor y mejor calidad en la adquisición de experiencias significativas entre los participantes. Aumentar los espacios temporales a la creación juvenil y la formulación de programas o proyectos que remedien o den soluciones a problemas reales del mundo de la vida.

Por otra parte, cabe indicar que resulta urgente reconocer en estos espacios de conocimiento y conversación una instancia para además de preparar dirigentes y líderes juveniles, para dar a conocer a la juventud el bagaje histórico y las luchas propias de anteriores dirigentes como muestra patente de la lógica continuidad-cambio tan presente en jornada como éstas.

10 Podestá: op. cit., p. 174. 
En esta misma línea, es imperioso comenzar, como muy pocas entidades en Chile lo hacen, generar textos juveniles de formación y reflexión, contextualizando dicho material a la región en la cual habitan los jóvenes participantes de jornadas o talleres de formación, recordando que en la diversidad ideológica se debe participar fomentando el diálogo y el respeto por otras ideas también sociales y preocupadas de la integridad del ser humano en sociedad.

La Araucanía, así como todas las otras regiones, requieren de juventud alegre y dinámica, pero también instruida en temáticas como la participación y el rol de la sociedad civil en el engranaje mundo público, social y privado. Hoy más que nunca, pues pareciera que por medio de la televisión, en horarios propios de la juventud, en literatura de dudosa monta o en sitios web, los jóvenes son entendidos como material moldeable, incapaz de asumir compromisos y envuelto en una especie de desgano vital.

En la novena región, este desafío presenta algunas variables que hacen de esta región un espacio geohistórico especial. Por una lado, la Araucanía es la región de Chile eminentemente pluriétnica y multicultural; es la tierra de los mapuche y también es la historia del colonialismo occidental, violento, usurpador y evangelizador; en ella se conjugan y coexisten el $\ddot{U}$ o canto mapuche y una inmensidad de tunas o clubes de cueca; de rukas y casas de corte europeo; de mapuduzgún o lengua mapuche, castellano, suizo, alemán o italiano; en fin, es una región compleja, por más que algunos actores - los empresarios del agro y forestales - nieguen tal cualidad y reduzcan la discusión acerca del desarrollo regional a las reivindicaciones territoriales de agrupaciones como el Consejo de Todas las Tierras o la Coordinadora Arauco-Malleco. Por otro lado, la novena región es el espacio geoadministrativo más pobre del país; lo avalan sus índices de indigencia, analfabetismo, deserción escolar, malos resultados en el SIMCE, cesantía y nimia inversión privada en este espacio.

Últimamente, estudios técnicos han comenzado a girar la visión en torno a este tema. Como ya se esbozó: para muchos la pobreza de la Araucanía iba de la mano del «problema mapuche», no obstante, informes de reciente data plantean algunas cuestiones estructurales que apuntan hacia la inversión y calidad de la infraestructura vial, portuaria, aérea, crediticia, tecnológica y científica, que no son controladas precisamente por la población mapuche sino que por el Estado, el sector privado y las universidades, principalmente, los responsables de la situación. Así la mirada a la Araucanía cambia sustancialmente y 
con ello debiesen cambiar las vías de solución, las propuestas y futuros programas de crecimiento y desarrollo integral de la región.

Por último, la juventud en general y la popular en particular, no detenta esquemas de participación y decisión en el seno de nuestro país. Las encuestas dicen que junto a mujeres e indígenas son los segmentos de población más excluidos. Deficiente nivel en la calidad de la educación media, nulo o mínimo acceso al sistema universitario, falta de oportunidades laborales y un gran cúmulo de sensaciones, frustraciones y falta de proyecciones constriñen el mundo de la vida, abortan las utopías y reducen los proyectos personales de estos sujetos. En la Araucanía esta juventud es un grito de espera, viven en una región irresuelta, «partida», en la cual unos (no-mapuche) no conocen a los otros (mapuche) y éstos interactúan en un espacio ajeno, en condiciones adversas y en nítida desventaja, lejos del otrora Lof Mapu, ${ }^{11}$ recíproco y correctamente normado por el Ad Mapu. ${ }^{12}$

ARAUCANÍA, VERANO 2003

11 Lof Mapu: familias del territorio; Lof Che: gente de la comunidad o territorio.

12 Ad Mapu: Leyes mapuche. 